\title{
The Roughness-Enhanced Light Emission from Metal-Oxide-Silicon Light-Emitting Diodes Using Very High Vacuum Prebake
}

\author{
Min-Hung Lee, Kuan-Fu Chen, Chang-Chi LAI, Chee Wee LiU*, Woei-Wu PAI ${ }^{1}$, \\ Miin-Jang CHEN ${ }^{2}$ and Ching-Fuh LIN ${ }^{2}$ \\ Department of Electrical Engineering and Graduate Institute of Electronics Engineering, National Taiwan University, Taipei, Taiwan, R.O.C. \\ ${ }^{1}$ Center for Condensed Matter Sciences, National Taiwan University, Taipei, Taiwan, R.O.C. \\ ${ }^{2}$ Department of Electrical Engineering and Graduate Institute of Electro-Optical Engineering, National Taiwan University, Taipei, Taiwan, R.O.C.
}

(Received November 5, 2001; accepted for publication December 20, 2001)

The oxide roughness of metal-oxide-silicon diodes can be intentionally controlled by the very high vacuum pre-bake and the growth conditions during rapid thermal oxidation. Both surface and $\mathrm{Si}$ /oxide interface have the similar magnitude of roughness measured by atomic force microscopy, indicating the conformal growth of oxide. At accumulation bias (positive gate bias), the holes tunnel from gate electrode to n-type Si through the ultrathin oxide, and recombine with the electrons in the accumulation region radiatively if phonon scattering and roughness scattering provide the necessary momentum. The light emission intensity increases with increasing oxide roughness. Strong electroluminescence with an external quantum efficiency of $\sim 2 \times 10^{-6}$ at room temperature was observed from a rough metal-oxide-silicon tunneling diode. [DOI: 10.1143/JJAP.41.L326]

KEYWORDS: metal oxide silicon diode, roughness, electroluminescence, electron-hole plasma recombination, external quantum efficiency

The Si-based optoelectronic devices attract great attention to process optical signal on the silicon chips $^{1)}$ with potential applications such as optical interconnects ${ }^{2)}$ and optical communication. Based on pn junctions, two different Si light emitters have been reported. A narrow-band infrared emitter with photon energy of silicon bandgap was implemented using a pn junction under forward bias with an external quantum efficiency of $\sim 10^{-4}$. $)$ Very recently, the band-edge emission from a simplified structure of pn junction with similar efficiency is also reported. ${ }^{4}$ Another broad (450-850 nm) visible light emitter was realized using an avalanche pn diode at reverse bias with the reported external quantum efficiency of $\sim 10^{-8}{ }^{3)}$ and $\left.\sim 10^{-6} .{ }^{5}\right)$ For metal-oxide-silicon (MOS) structures, both the infrared band-edge emission ${ }^{2)}$ and the broad visible emission ${ }^{6}$ are also observed at accumulation bias. Note that the same MOS tunneling structure biased at deep depletion region can be also used as a photodetector. ${ }^{7)}$ Although there is a concern of the reliability of utrathin oxide in the MOS structure, the incorporation of deuterium into the oxide has been demonstrated to improve the degradation of light emission intensity. ${ }^{8)}$ Due to the indirect bandgap nature of $\mathrm{Si}$, additional momentum is required for the light emission process. The phonon scattering ${ }^{9)}$ and the oxide roughness scattering ${ }^{10)}$ can provide the additional momentum for the radiative electron-hole recombination at oxide/Si interface. In this letter, an effective way to increase the oxide roughness by very high vacuum pre-bake before rapid thermal oxidation is demonstrated. The light emission intensity increases with the magnitude of oxide roughness up to the external quantum efficiency of $2 \times 10^{-6}$ for the MOS light-emitting diode (LED).

The ultrathin gate oxide was grown by rapid thermal oxidation on $1-10 \Omega$-cm n-type wafers at the $800-900^{\circ} \mathrm{C}$. The gas flows were $500 \mathrm{sccm}$ nitrogen and $500 \mathrm{sccm}$ oxygen at a reduced pressure. After a HF dip, the wafer was transferred to the process chamber through the load-lock chamber, and was in situ baked in the very high vacuum $\left(<3 \times 10^{-6}\right.$ Torr, maintained by a turbo pump) at $1000^{\circ} \mathrm{C}$, and then, the wafer

\footnotetext{
*Corresponding author. E-mail address: chee@cc.ee.ntu.edu.tw
}

was baked in hydrogen at $1000^{\circ} \mathrm{C}$ for $1 \mathrm{~min}$. Note that this very high vacuum bake should yield a rough and clean Si surface. ${ }^{11,12)}$ The oxide growth was conducted after the hydrogen bake, and subsequently nitrogen post-oxide-anneal was performed at $900^{\circ} \mathrm{C}$ for $10 \mathrm{~min}$. The oxide thickness is measured by ellipsometry. The roughness is measured by atomic force microscopy (AFM) and its magnitude is defined as the root-mean-square of height variation. The surface roughness on the oxide is measured right after the oxide growth, and the $\mathrm{Si} /$ oxide interface roughness is measured immediately after the oxide removal of the same samples by HF dip. The ellipsometry confirms little native oxide growth after AFM measurements for both conditions. The surface roughness and interface roughness have similar magnitude (Fig. 1). This indicates the conformal growth of the rapid thermal oxidation. ${ }^{13)}$ Note that our AFM can not resolve the spatial spectrum variation of these samples. However, ultrathin thermal oxide has similar bandwidth in the interface roughness spectra as measured by atomic force microscopy(AFM) and mobility measurement. ${ }^{14)}$ The PMOS (p-channel) LED has Al gate electrodes with circular areas defined by photolithography. An-

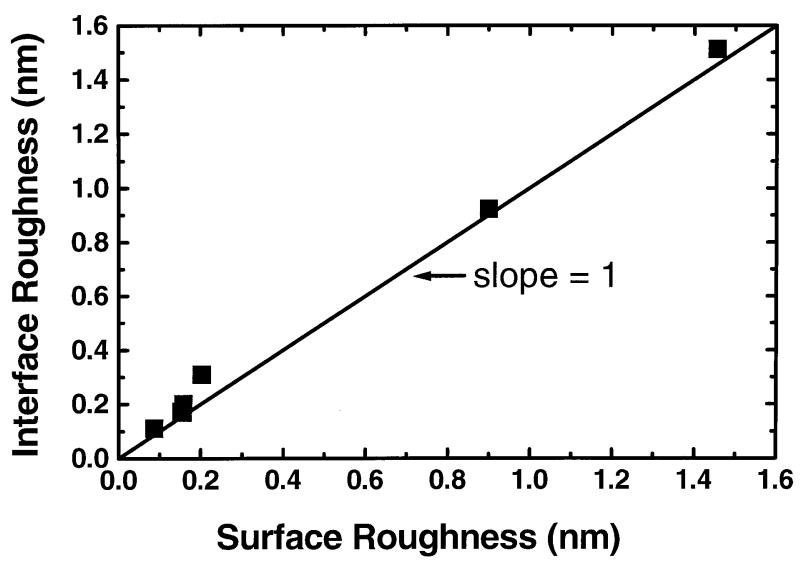

Fig. 1. The plot of surface oxide roughness vs interface roughness between $\mathrm{Si} /$ oxide. The linear relationship indicates the conformal growth of rapid thermal oxidation. 


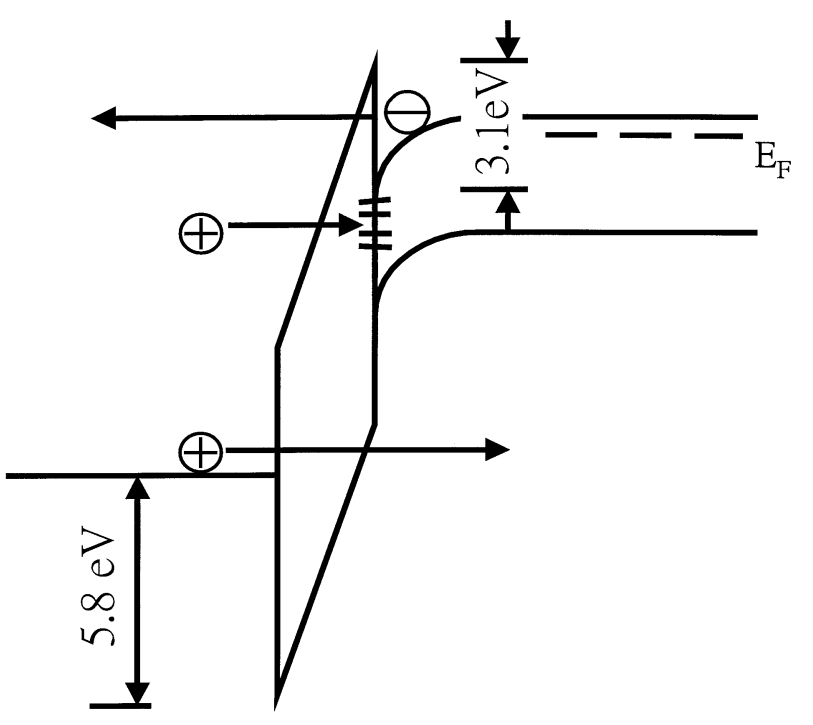

Al Oxide n-Si

Fig. 2. The current components of a PMOS tunneling diode. The hole current is smaller than the electron current. The hole current tunneling to the interface states cannot contribute the radiative recombination. Neither does the electron current component.

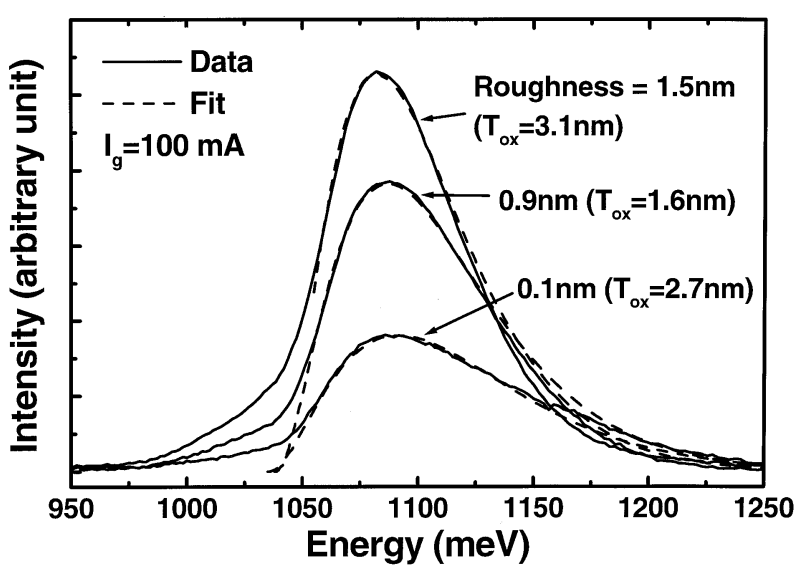

Fig. 3. The EL spectra of PMOS tunneling diodes with different oxide roughness. The oxide thickness and voltage are indicated in the parentheses.

other $\mathrm{Al}$ contact is on the back of the wafer.

At the positive gate bias, the holes in the $\mathrm{Al}$ electrode tunnel to the n-Si through the ultrathin oxide, and meanwhile the electrons in the accumulation region of $\mathrm{n}$-Si tunnel to the $\mathrm{Al}$ electrode (Fig. 2). Note that the holes tunneling to the interface states as shown in the Fig. 2 cannot contribute the radiative band-edge recombination. Due to barrier height difference $\left(3.1 \mathrm{eV}\right.$ for electrons and $5.8 \mathrm{eV}$ for holes $\left.{ }^{15,16)}\right)$, the hole tunneling current of PMOS devices is smaller than the electron tunneling current. The tunneling holes from Al to $\mathrm{n}$-Si in PMOS can be scattered by oxide roughness and radiatively recombine with electrons in the accumulation layer with the assistance of phonons. ${ }^{10)}$ Figure 3 shows the electroluminescence from the PMOS devices with the device area of $4 \times 10^{-2} \mathrm{~cm}^{2}$ for different oxide roughness. The light emission of the $\mathrm{Al}$ gate MOS devices is collected from the edge of the gate electrode at the drive current of $100 \mathrm{~mA}$. All the emission spectra can be fitted by the electron-hole-

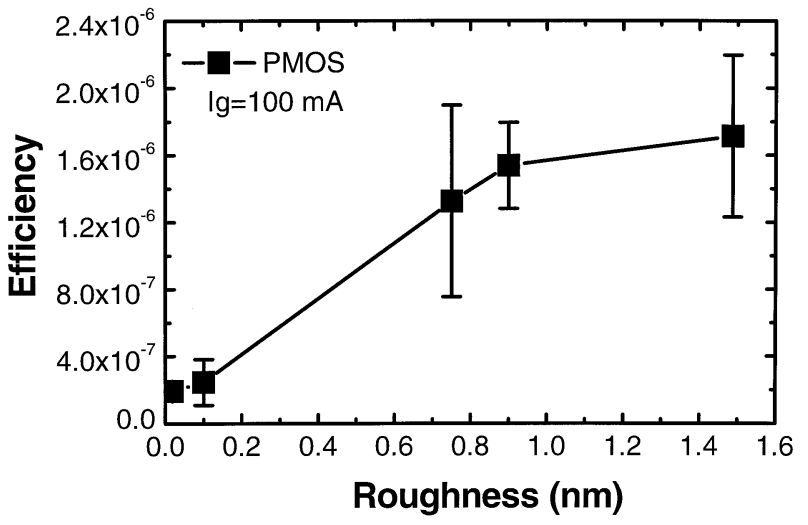

Fig. 4. The external quantum efficiency vs oxide roughness. The error bar is the standard deviation of external quantum efficiency for a set of devices.

plasma recombination model, which is the convolution between electron and hole populations by considering the energy conservation. ${ }^{17)}$ Note that the surface roughness and the interface roughness have similar magnitude due to the conformal growth of the oxide. The emission intensity of the MOS devices increases with the oxide roughness, while the oxide thickness of each device is different. Both the phonon scattering and oxide roughness scattering can conserve the momentum during the electron-hole radiative recombination at oxide/Si interface, but at a fixed device temperature, the phonon population should be constant and oxide roughness is the main factor to affect the emission efficiency. Note that the similar line widths in the electroluminescence spectra (Fig. 3) indicate the similar device temperatures. ${ }^{9)}$ To confirm this observation, a set of PMOS devices have been measured for each oxide roughness, and the statistical results of external quantum efficiency at the current of $100 \mathrm{~mA}$ vs oxide roughness are shown in Fig. 4. The error bar in Fig. 4 is the standard deviation of the device set for efficiency measurements. Although there are some variations of external quantum efficiency, the trend definitively shows that the efficiency increases with increasing roughness. The spectral distribution of roughness cannot be accurately measured in our AFM due to the lateral resolution of the AFM tips. In the litrature, ${ }^{14)}$ the spectral distribution of the roughness have no consistent results between AFM measurement and mobility measurement, but the spectral distributions are similar for different roughness magnitudes in both measurements. It is reasonable to assume that the spatial spectra in our samples are similar and the spectral distribution of oxide roughness has smaller effect on the enhancement of emission intensity than the magnitude of the roughness.

A particular PMOS device with oxide roughness of $1.5 \mathrm{~nm}$ shows an external quantum efficiency of $2.5 \times 10^{-6}$ at a $100 \mathrm{~mA}$, and the efficiency increases as drive current increases (Fig. 5). Based on the model calculation ${ }^{18)}$ of tunneling current (Fig. 2), the ratio of hole current to electron current increases with large gate bias, and only the hole tunneling current can contribute the light emission. This may be responsible for the increase of emission efficiency with the increasing gate current.

We have demonstrated that the correlation between oxide roughness and electroluminescence intensity in the PMOS tunneling diodes. The high vacuum pre-bake seems to be an 


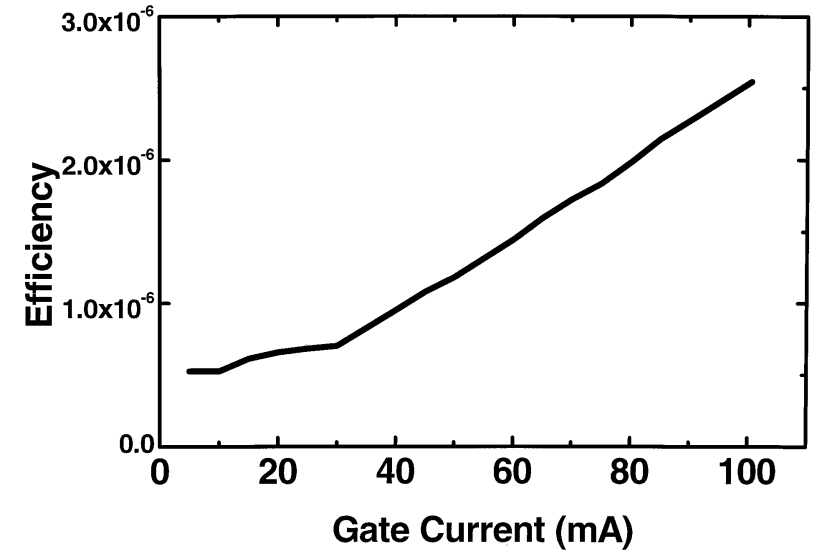

Fig. 5. The external quantum efficiency vs drive current of a particular PMOS LED.

effective method to produce oxide roughness, and the MOS LED emits more light after such very high vacuum pre-bake process.

This work is supported by TSMC and National Science Council, Taiwan, R.O.C. (89-2218-E-002-017, 89-2218-E002-054).

1) K. D. Hirschman, L. Tysbekov, S. P. Duttagupta and P. M. Fauchet: Nature 384 (1996) 338.
2) C. W.Liu, M. H. Lee, C. F. Lin, I. C. Lin, W. T. Liu and H. H. Lin: Int. Electron Devices Meet. Tech. Dig., 1999, Washington D.C., p. 749.

3) J. Kramer, P. Seitz, E. F. Steigmeier, H. Auderset and B. Delley: Sens. \& Actuat. A 37/38 (1993) 527.

4) W. L. Ng, M. A. Lourenco, R. M. Gwilliam, S. Ledain, G. Shao and K. P. Homewood: Nature 410 (2001) 192.

5) L. W. Snymandu, M. Plessis, E. Seevinck and H. Aharoni: IEEE Electron Device Lett. 20 (1999) 614.

6) C. W. Liu, M. H. Lee, S. T. Chang, W. T. Liu, M.-J. Chen and C.-F. Lin: Appl. Phys. Lett. 77 (2000) 4347.

7) C. W. Liu, W. T. Liu, M. H. Lee, W. S. Kuo and B.-C. Hsu: IEEE Electron Device Lett. 21 (2000) 307.

8) C. W. Liu, C.-H. Lin, M. H. Lee, S. T. Chang, Y. H. Liu, M.-J. Chen and C.-F. Lin: Appl. Phys. Lett. 78 (2001) 1397.

9) C. W. Liu, M.-J. Chen, I. C. Lin, M. H. Lee and C.-F. Lin: Appl. Phys. Lett. 77 (2000) 1111.

10) C. W. Liu, M. H. Lee, M.-J. Chen, C.-F. Lin and M. Y. Chern: IEEE Electron Device Lett. 21 (2000) 601.

11) X. Xu, R. T. Kuehn, M. C. Ozturk, J. J. Wortman, R. J. Nemanich, G. S. Harris and D. M. Maher: J. Electronic Mater. 22 (1993) 335.

12) F. W. Smith and G. Ghidini: J. Electrochem. Soc. 129 (1982) 1300

13) V. Tsai, X.-S. Wang, E. D. Williams, J. Schneir and R. Dixson: Appl. Phys. Lett. 71 (1997) 1495.

14) A. Pirovano, A. L. Lacaita, G. Ghidini and G. Tallarida: IEEE Electron Device Lett. 21 (2000) 34.

15) W.-C. Lee and C. Hu: VLSI Tech. Dig., 2000, Hawaii, p. 198.

16) Y. Taur and T. H. Ning: Fundamentals of Modern VLSI Devices (Cambridge University Press, Cambridge, 1998) p. 59.

17) C. W. Liu, M. H. Lee, M.-J. Chen, I. C. Lin and C.-F. Lin: Appl. Phys. Lett. 76 (2000) 1516.

18) S. Keeney, F. Piccini, M. Morelli, A. Mathewson, C. Lombardi, R. Bez, L, Ravazzi and D. Cantarelli: Int. Electron Devices Meet. Tech. Dig., 1990, San Francisco, p. 201. 